\title{
Zero-temperature limit of thermodynamic quantum master equations
}

\author{
Hans Christian Öttinger* \\ ETH Zürich, Department of Materials, Polymer Physics, HCP F 47.2, CH-8093 Zürich, Switzerland
}

(Received 29 November 2017; published 23 July 2018)

\begin{abstract}
We investigate the zero-temperature limit of thermodynamic quantum master equations that govern the time evolution of density matrices for dissipative quantum systems. The quantum master equations for $T=0$ and for $T>0$ possess completely different structures: (i) the equation for $T=0$ is linear in the deviation from the ground-state density matrix, whereas the equation for $T>0$, in general, is seriously nonlinear, and (ii) the Gibbs state is obtained as the steady-state solution of the nonlinear equation for $T>0$, whereas the ground state cannot be found from the equation for $T=0$. Nevertheless, the equation for $T=0$ can reproduce the behavior for $T \gtrsim 0$ remarkably well. We discuss some implications of that observation for dissipative quantum field theory.
\end{abstract}

DOI: 10.1103/PhysRevA.98.012131

\section{INTRODUCTION}

Understanding and controlling quantum dissipation is a key problem in developing a variety of modern quantum technologies. A frequently used tool for describing dissipative quantum systems is quantum master equations [1,2], the most popular of which are the Lindblad master equations [3] based on linearity and complete positivity assumptions. As an alternative option, the principles of nonequilibrium thermodynamics may be used to derive quantum master equations. The emergence of irreversibility from reversible equations is a highly nontrivial and controversial topic, and it is hard to believe that this should be any easier for quantum than for classical systems. We hence prefer to rely on the quantization of the equations of classical nonequilibrium thermodynamics [4,5], for which there exists a highly developed framework possessing a welldefined mathematical structure [6-12]. The final step of the development of thermodynamic quantum master equations has been made in [5], where the setting has been generalized so that the linear Davies Lindblad master equations [13] are contained as a special class of thermodynamic master equations. It has been shown in [14] that the nonlinear quantum master equations proposed in [5] have the potential to explain ultralong coherence of a qubit.

A characteristic feature of thermodynamic quantum master equations is that, in general, they can be nonlinear in the density matrix, contrary to one of the basic assumptions of the Lindblad theory. Various implications of the thermodynamic approach, including nonlinearity, have been discussed over the last 25 years [15-21]. As nonlinear equations are much more difficult to handle than linear ones [22], we here investigate possible simplifications resulting in the zero-temperature limit of thermodynamic quantum master equations. More concretely, we

\footnotetext{
*hco@mat.ethz.ch; http://www.polyphys.mat.ethz.ch/

Published by the American Physical Society under the terms of the Creative Commons Attribution 4.0 International license. Further distribution of this work must maintain attribution to the author(s) and the published article's title, journal citation, and DOI.
}

are motivated by the relevance of zero-temperature quantum master equations to dissipative quantum field theory [23].

After presenting the general thermodynamic and zerotemperature quantum master equations in the context of a two-level system (Sec. II), a detailed comparison of these equations for different types of initial conditions is carried out (Sec. III). The zero-temperature equation requires separate knowledge of the ground state that can be obtained by solving the Bloch equation (Sec. IV). A brief summary and a detailed discussion conclude the paper (Sec. V).

\section{THERMODYNAMIC QUANTUM MASTER EQUATIONS}

We consider thermodynamic quantum master equations for systems in which the full Hamiltonian $H$ can be written as the sum $H=H^{\text {free }}+H^{\text {coll }}$ of free and collisional (or interaction) contributions. The quantum master equation for a free system is assumed to be of the linear Lindblad form,

$$
\begin{aligned}
\frac{d \rho_{t}}{d t}= & -i\left[H^{\text {free }}, \rho_{t}\right]+\gamma\left(2 A \rho_{t} A^{\dagger}-\left\{A^{\dagger} A, \rho_{t}\right\}\right. \\
& \left.+e^{-2 \beta}\left(2 A^{\dagger} \rho_{t} A-\left\{A A^{\dagger}, \rho_{t}\right\}\right)\right),
\end{aligned}
$$

where we assume single forward and backward dissipative processes characterized by the coupling operators $A, A^{\dagger}$ and the relaxation rate $\gamma$ (the generalization to several dissipation processes is straightforward) and an associated energy gap of 2 (thus fixing the units of energy). The commutator and anticommutator of two operators are indicated by square and curly brackets, respectively, $\hbar=1$ is assumed for convenience, the parameter $\beta=1 /\left(k_{\mathrm{B}} T\right)$ is the inverse temperature of the heat bath surrounding the quantum system, and the factor $e^{-2 \beta}$, which is introduced to obtain convergence to the proper Gibbs state, requires further consideration of the specific situation considered here. With these conventions, the units of time are given by $t_{0}=2 / \omega_{0}$, where $\omega_{0}$ is the frequency associated with the energy gap (the oscillation period in units of $t_{0}$ is given by $\pi$ ).

It is convenient to work in the eigenbasis of the free Hamiltonian, so that $H^{\text {free }}$ is diagonal. If, for illustrative purposes, we restrict ourselves to the simplest example of 
a two-level system, then, without loss of generality, we can assume

$$
H^{\text {free }}=\left(\begin{array}{ll}
2 & 0 \\
0 & 0
\end{array}\right),
$$

where we have shifted the ground-state energy to zero and chosen units of energy such that the energy gap has the value 2 . The operators

$$
A=\left(\begin{array}{ll}
0 & 0 \\
1 & 0
\end{array}\right), \quad A^{\dagger}=\left(\begin{array}{ll}
0 & 1 \\
0 & 0
\end{array}\right),
$$

are chosen such that they take us from the excited state to the ground state and vice versa. The energy gap of 2 between the two states motivates the factor $e^{-2 \beta}$ between the two rates in the quantum master equation (1). The commutation relations

$$
\left[H^{\text {free }}, A^{\dagger}\right]=2 A^{\dagger}, \quad\left[H^{\text {free }}, A\right]=-2 A,
$$

express the essential algebraic structure of the free two-level system.

The most general collisional Hamiltonian in the energy base of the free theory is given by the off-diagonal matrix

$$
H^{\text {coll }}=\left(\begin{array}{cc}
0 & \lambda \\
\lambda^{*} & 0
\end{array}\right) .
$$

Throughout this paper, we assume that the interaction strength $\lambda$ is a real parameter, that is, $\lambda^{*}=\lambda$. The energies of the full Hamiltonian are given by $1 \pm \sqrt{1+\lambda^{2}}$, so that the energy gap of the interacting theory becomes $2 \sqrt{1+\lambda^{2}}>2$.

According to Eq. (1.57) of [23], the quantum master equation (1) of the free system can be extended to an equation for the interacting theory (see Appendix A for details),

$$
\begin{aligned}
\frac{d \rho_{t}}{d t}= & -i\left[H, \rho_{t}\right]+\gamma\left(2 A \rho_{t} A^{\dagger}-\left\{A^{\dagger} A, \rho_{t}\right\}\right. \\
& \left.+e^{-2 \beta}\left(2 A^{\dagger} \rho_{t} A-\left\{A A^{\dagger}, \rho_{t}\right\}\right)\right) \\
& -\beta \gamma \int_{0}^{1} e^{-2 u \beta}\left(\left[A, \rho_{t}^{1-u}\left[A^{\dagger}, H^{\mathrm{coll}}\right] \rho_{t}^{u}\right]\right. \\
& \left.+\left[A^{\dagger}, \rho_{t}^{u}\left[A, H^{\mathrm{coll}}\right] \rho_{t}^{1-u}\right]\right) d u .
\end{aligned}
$$

The steady-state solution of this equation is given by the Gibbs state of the interacting system,

$$
\rho_{\mathrm{eq}}=\frac{e^{-\beta H}}{\operatorname{tr}\left(e^{-\beta H}\right)},
$$

and the underlying entropy is of the von Neumann form. Note that the splitting of the density matrix into the two factors $\rho_{t}^{u}$ and $\rho_{t}^{1-u}$ in the collision part of Eq. (6) leads to an additively nonlinear dependence of this quantum master equation on $\rho_{t}$. For the two-level system, the integrand in Eq. (6) can be simplified by using the commutators

$$
\left[A^{\dagger}, H^{\text {coll }}\right]=\lambda \sigma_{3}, \quad\left[A, H^{\text {coll }}\right]=-\lambda \sigma_{3},
$$

where $\sigma_{3}$ is the Pauli matrix

$$
\sigma_{3}=\left(\begin{array}{cc}
1 & 0 \\
0 & -1
\end{array}\right)
$$

Note that in terms of this Pauli matrix we have $H^{\text {free }}=\sigma_{3}+1$.

We now turn to the zero-temperature limit of the quantum master equations for the free and interacting theories. For the free theory, we only need to omit the term suppressed by the factor $e^{-2 \beta}$ in Eq. (1). For the interacting theory, the naive zero-temperature limit obtained by replacing $2 \beta e^{-2 u \beta}$ for $\beta \rightarrow \infty$ by $\delta(u)$ leads to a linear quantum master equation that does not converge to the proper ground state [23]. If one first linearizes the master equation around the Gibbs state and then performs the zero-temperature limit, one obtains the following more appropriate quantum master equation (see Eq. (1.65) of [23]):

$$
\begin{aligned}
\frac{d \Delta \rho_{t}}{d t}= & -i\left[H, \Delta \rho_{t}\right]+\gamma\left(2 A \Delta \rho_{t} A^{\dagger}-\left\{A^{\dagger} A, \Delta \rho_{t}\right\}\right) \\
& -\frac{\gamma}{2}\left(\left[A, \Delta \rho_{t}\left[A^{\dagger}, H^{\text {coll }}\right]\right]+\left[A^{\dagger},\left[A, H^{\text {coll }}\right] \Delta \rho_{t}\right]\right),
\end{aligned}
$$

where $\Delta \rho_{t}=\rho_{t}-\rho_{\text {ground }}$ is the deviation from the density matrix characterizing the ground state of the interacting system, that is,

$$
\rho_{\text {ground }}=\frac{1}{2(1+\kappa)}\left(\begin{array}{cc}
\kappa & -\lambda \\
-\lambda & 2+\kappa
\end{array}\right),
$$

and the relation between $\lambda$ and $\kappa$ is defined by the equation $(1+\kappa)^{2}=1+\lambda^{2}$. By means of the commutators (8), the zerotemperature quantum master equation (10) for our two-level system becomes

$$
\begin{aligned}
\frac{d \Delta \rho_{t}}{d t}= & -i\left[H, \Delta \rho_{t}\right]+\gamma\left(2 A \Delta \rho_{t} A^{\dagger}\right. \\
& \left.-\left\{A^{\dagger} A, \Delta \rho_{t}\right\}\right)-\frac{\gamma \lambda}{2}\left(A \Delta \rho_{t} \sigma_{3}\right. \\
& \left.+\sigma_{3} \Delta \rho_{t} A^{\dagger}-\Delta \rho_{t} \sigma_{3} A-A^{\dagger} \sigma_{3} \Delta \rho_{t}\right) .
\end{aligned}
$$

Generalization of Eqs. (10) and (12) to multiple dissipation modes is straightforward. Whereas the quantum master equation (6) at finite temperatures is nonlinear, the zerotemperature limit (12) is linear in the deviation from the ground-state density matrix. The exponential detailed-balance factors required in Eq. (6) to find the equilibrium state (7) have disappeared in the zero-temperature limit so that the ground state cannot be found by Eq. (10) or (12) and is required as a separate input. This change of structure leads to an enormous simplification. The eigenvalues of the linear system (12) imply that all elements of the matrix $\Delta \rho_{t}$ obtained by solving the zero-temperature quantum master equation are linear combinations of the three functions

$$
e^{-2 \gamma t}, \quad \cos \left(\omega_{\lambda} t\right) e^{-\gamma t}, \quad \sin \left(\omega_{\lambda} t\right) e^{-\gamma t},
$$

where $\omega_{\lambda}=2 \sqrt{1+\lambda^{2}}$ is the energy gap of the interacting system. In the following, we do not make any use of closedform solutions of master equations.

\section{NUMERICAL RESULTS}

We now compare the solutions of the zero-temperature quantum master equation (12) and the solutions of the nonlinear quantum master equation (6) at low temperatures. We solve both equations numerically by the ode 45 routine of MATLAB, which is a six-stage, fifth-order Runge-Kutta method. 


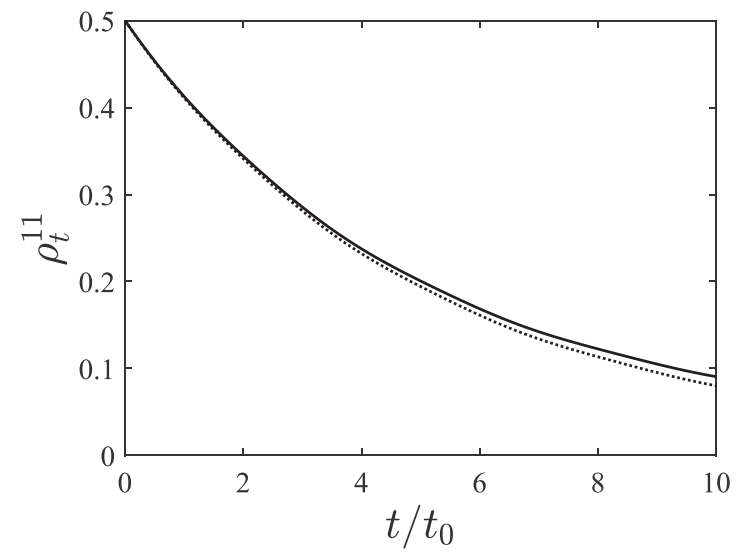

FIG. 1. Decay of the diagonal density-matrix element $\rho_{t}^{11}$ in the approach to the steady state. The initial density matrix is taken as one-half of the unit matrix, which corresponds to the high-temperature limit of a two-level system. The continuous curve is for $T=0.5$, the dotted curve is for $T=0$. The further parameters are $\lambda=0.25$ and $\gamma=0.1$.

After diagonalization of the $2 \times 2$ matrix $\rho_{t}$, the integral in Eq. (6) can readily be evaluated in closed form (for example, by the MATLAB routine int). Throughout this section we use the interaction strength $\lambda=0.25$ and the small relaxation rate $\gamma=0.1$, so that the temperature $T$ (or its inverse $\beta$ ) is the only remaining parameter.

To present a density matrix $\rho_{t}$ for the two-level system, we show either $\rho_{t}^{11}$ or $\rho_{t}^{00}$ (with $0 \leqslant \rho_{t}^{00} \leqslant 1,0 \leqslant \rho_{t}^{11} \leqslant 1$ ) because these diagonal density-matrix elements are related by $\rho_{t}^{00}+\rho_{t}^{11}=1$. In view of the self-adjointness of $\rho_{t}$, a complete representation of the density matrix is then achieved by additionally displaying the real and imaginary parts of the off-diagonal matrix element $\rho_{t}^{10}$.

We first consider the relaxation of the high-temperature initial density matrix, that is, one-half of the unit matrix, to equilibrium. Figure 1 shows the decay of the diagonal element $\rho_{t}^{11}$. Even for $T=1 / 2$, the solution of the nonlinear quantum master equation (6) is very close to the result obtained from the zero-temperature equation (12), where the exact ground state (11) has been used to reconstruct $\rho_{t}$ from $\Delta \rho_{t}$. For the off-diagonal matrix element $\rho_{t}^{10}$, the imaginary parts in Fig. 2 are almost indistinguishable, and also the real parts for $T=0$ and $T=1 / 2$ are remarkably close. This observation is particularly remarkable, as the high-temperature initial condition is far away from the ground state. Figure 3 shows that, for larger times, the oscillations around the steady-state values decay slowly. The zero-temperature quantum master equation describes the relaxation behavior of the density matrix for a considerable range of temperatures and for initial conditions far from the ground state. As a consequence, the matrix elements at low temperatures can be approximated by a superposition of exponential decay and damped oscillations according to Eq. (13).

The high-temperature initial condition considered so far has the advantage of being easily realizable. If we use the excited state of the free or interacting system as an even more extreme initial condition, the relaxation of the density matrix looks

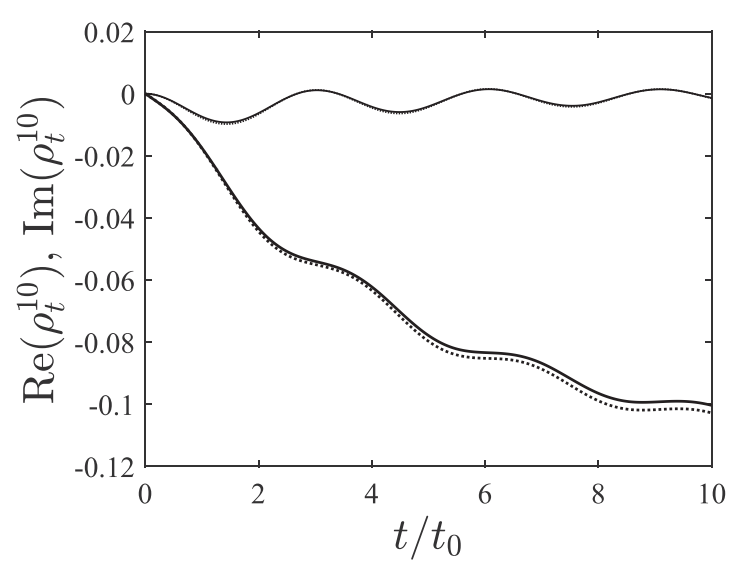

FIG. 2. Real part (lower curves) and imaginary part (upper curves) of the off-diagonal density-matrix element $\rho_{t}^{10}$ in the approach to the steady state. The initial density matrix is taken as one-half of the unit matrix, which corresponds to the high-temperature limit of a two-level system. Continuous curves are for $T=0.5$, dotted curves are for $T=0$. The further parameters are $\lambda=0.25$ and $\gamma=0.1$.

similar. As an alternative initial condition we hence look at

$$
\Delta \rho_{0}=\alpha\left(\begin{array}{ll}
0 & 1 \\
1 & 0
\end{array}\right),
$$

where $\Delta \rho_{t}$ is the deviation from the ground-state density matrix for $T=0$ and from the Gibbs state for finite temperature $T ; \alpha$ is an amplitude coefficient.

The corresponding solutions of the nonlinear thermodynamic and of the zero-temperature quantum master equations for $\alpha=0.1$ are displayed in Figs. 4 and 5. The diagonal deviatoric density-matrix element $\Delta \rho_{t}^{11}$ in Fig. 4 now starts at zero and, with damped oscillations, returns to zero. Even for $T=1$, the deviation from the zero-temperature result is very small. In view of the initial condition (14), the real and imaginary parts of $\Delta \rho_{t}^{10}$ in Fig. 5 start at $\alpha=0.1$ and 0 , respectively, and approach zero by damped oscillations. The frequency $\omega_{\lambda}$ of the oscillations is increased by the interactions, and the damping rate is given by the small parameter $\gamma$. The off-diagonal matrix element $\Delta \rho_{t}^{10}$ is closely related to a correlation function or to a propagator in quantum field

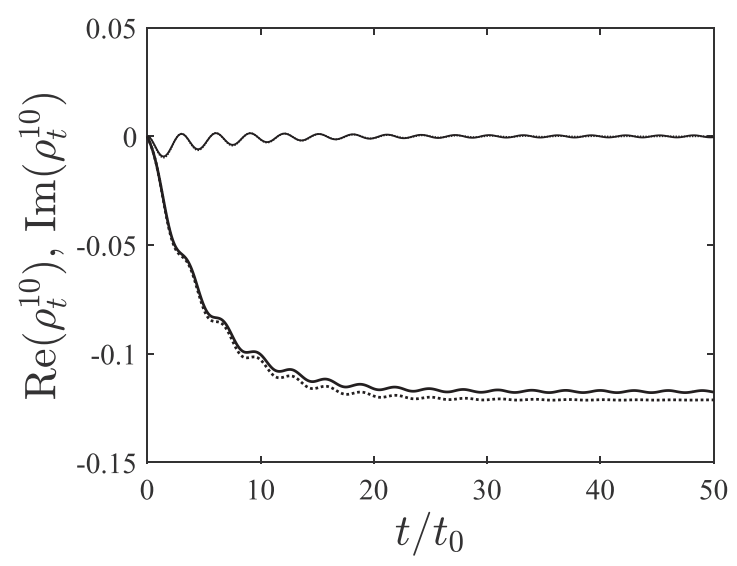

FIG. 3. Same as Fig. 2, but for larger times. 


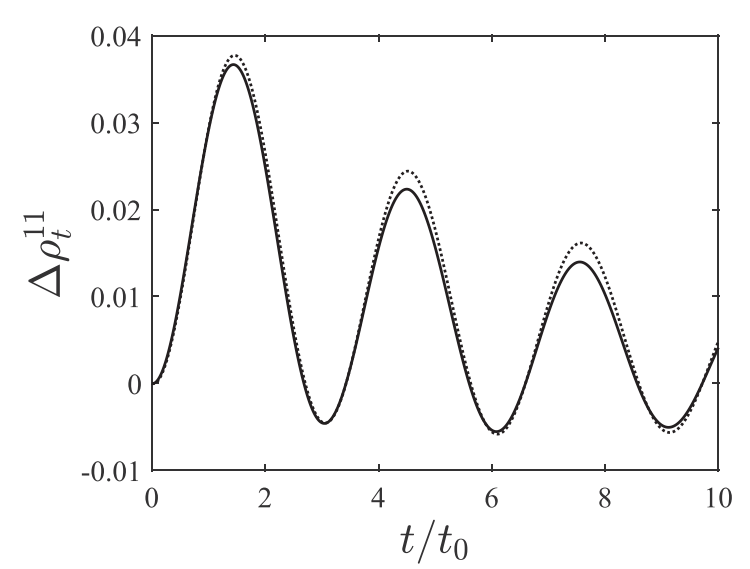

FIG. 4. Damped oscillations of the deviatoric density-matrix element $\Delta \rho_{t}^{11}$ in the approach to the steady state. The off-diagonal initial condition is of the form (14) with $\alpha=0.1$. The continuous curve is for $T=1.0$, the dotted curve is for $T=0$. The further parameters are $\lambda=0.25$ and $\gamma=0.1$.

theory. More generally, the calculation of correlation functions $[1,23,24]$ corresponds to traceless but non-self-adjoint $\Delta \rho_{0}$.

\section{BLOCH EQUATION FOR THE GROUND STATE}

The quantum master equation (12) can be used to calculate the deviatoric density matrix $\Delta \rho_{t}$. To find the full density matrix $\rho_{t}=\rho_{\text {ground }}+\Delta \rho_{t}$ we need separate knowledge of the ground-state density matrix. However, $\rho_{\text {ground }}$ is not known in general. It can be found without diagonalizing the Hamiltonian by solving the Bloch equation in the limit $\tau \rightarrow \infty$,

$$
\frac{d \rho_{\tau}}{d \tau}=-\frac{1}{2}\left(H \rho_{\tau}+\rho_{\tau} H\right)+\operatorname{tr}\left(H \rho_{\tau}\right) \rho_{\tau} .
$$

As the units of energy are fixed in terms of the gap width of the free Hamiltonian, the parameter $\tau$ is dimensionless. The Bloch equation can formally be considered as a reversible quantum master equation in imaginary time, but it is simply written down such that it has the Gibbs distribution as its solution. The

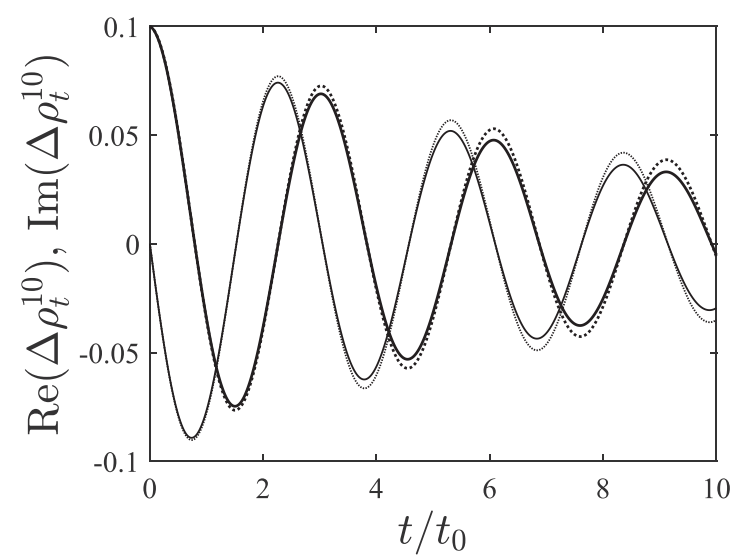

FIG. 5. Real part (thick curves) and imaginary part (thin curves) of the deviatoric density-matrix element $\Delta \rho_{t}^{10}$ in the approach to the steady state. The off-diagonal initial condition is of the form (14) with $\alpha=0.1$. Continuous curves are for $T=1.0$, dotted curves are for $T=0$. The further parameters are $\lambda=0.25$ and $\gamma=0.1$.

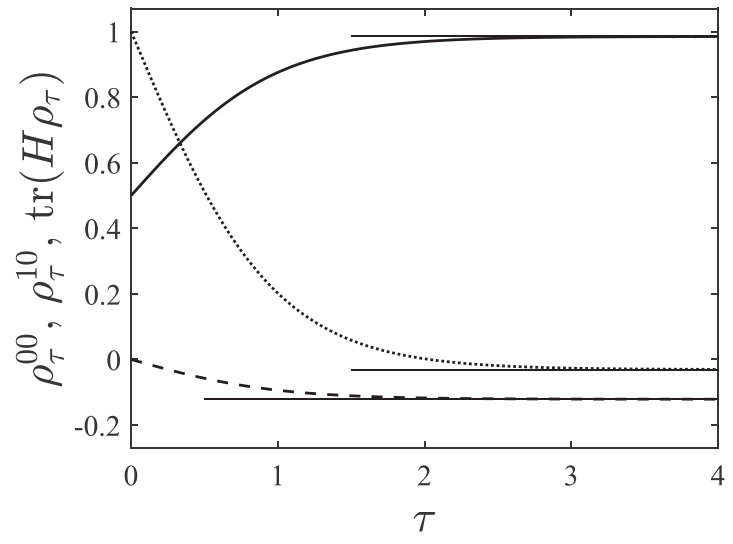

FIG. 6. Solution of the Bloch equation (15) for $\lambda=0.25$. The initial density matrix is one-half of the unit matrix, which corresponds to the high-temperature limit of a two-level system. In addition to the matrix elements $\rho_{t}^{00}$ (continuous curve) and $\rho_{t}^{10}$ (dashed curve), the decay of the average energy (dotted curve) is shown. The exact results for the ground state are indicated by the thin horizontal lines.

practical usefulness of the Bloch equation is underlined by the fact that it is the basis for the density-matrix quantum Monte Carlo method [25,26]. Assuming a finite number of degrees of freedom, its exact solution for the high-temperature initial condition is indeed given by

$$
\rho_{\tau}=\frac{e^{-\tau H}}{\operatorname{tr}\left(e^{-\tau H}\right)}
$$

The solution for the two-level system with interaction strength $\lambda=0.25$ is shown in Fig. 6. As before, all curves have been calculated with the ode 45 routine of MATLAB rather than by diagonalizing the Hamiltonian in the explicit solution (16). The diagonal matrix element $\rho_{t}^{00}$ increases from its initial value $1 / 2$ to a steady-state value close to 1 (continuous curve). The off-diagonal matrix element $\rho_{t}^{10}$ starts off at zero, remains real, and converges to a small negative value (dashed curve). As additional information, the decay of the average energy from the initial high-temperature value 1 to the negative ground-state value $1-\sqrt{1+\lambda^{2}}$ is shown by the dotted line in Fig. 6. For $\tau=4$, all deviations from the exact results for the ground state (indicated by the thin horizontal lines in Fig. 6) are less than $2 \%$. Numerical integration of the Bloch equation indeed is a tool for calculating the ground state in the limit $\tau \rightarrow \infty$. According to Eq. (16), the results at finite $\tau$ represent the Gibbs state (7) at finite inverse temperature $\beta=\tau$. In the density-matrix quantum Monte Carlo method [25,26], the numerical integration of the Bloch equation is performed with the explicit Euler scheme.

\section{SUMMARY AND DISCUSSION}

A proper zero-temperature quantum master equation is obtained by first linearizing a thermodynamic quantum master equation and then passing to the limit of vanishing temperature. In spite of a complete change in structure from nonlinear in the density matrix to linear in its deviation from the ground state, the zero-temperature approximation for a two-level 
system works remarkably well, even at unexpectedly high temperatures (of the order of the energy gap).

The zero-temperature limit is performed for a constant rate parameter characterizing the strength of dissipation. This assumption is consistent with the fluctuation-dissipation theorem [27] in the sense that the steady state of the thermodynamic quantum master equation is the Gibbs state (7) at arbitrarily small temperatures $T>0$. At $T=0$, however, the ground state cannot be found from the limit master equation. This is a consequence of the loss of detailed balance at zero temperature. The ground state requires a separate calculation, for example, based on the Bloch equation. A constant rate parameter may be particularly appealing if dissipation does not arise as an emergent phenomenon but is considered as a fundamental feature of nature. This idea of fundamental dissipation might lead to an alternative to string theory or loop quantum gravity as a framework for understanding quantum gravity. This fundamental dissipation would lead to unresolvable clouds of free particles on the Planck scale. Dissipation hence offers a simple mechanism for putting a lower limit to physically resolvable length scales (for more details, see pp. 28 and 228 of [23]).

Solving the linear zero-temperature quantum master equation (12) is a much easier task than solving the nonlinear thermodynamic quantum master equation (6) at low temperatures. This remark holds both for analytical calculations (such as perturbation theory) and for numerical techniques (such as stochastic simulations). Moreover, much more general correlation functions can be defined in a linear setting than for nonlinear quantum master equations $[1,23,24]$.

Quantum master equations provide an ideal starting point for stochastic simulation techniques. Unravelings, in which the evolution of density matrices is obtained from suitably constructed stochastic processes in Hilbert space, are a fundamental tool for studying the solutions of linear [1] and nonlinear $[28,29]$ quantum master equations. The linear zerotemperature quantum master equation (12) is easy to simulate. However, it requires a two-process unraveling because the trace of $\Delta \rho_{t}$ vanishes. A two-process unraveling is also advantageous because $\Delta \rho_{t}$ is multiplied by different matrices from the left and right in the interaction term of Eq. (12). The density-matrix quantum Monte Carlo method [25,26] for finding the ground state is an ideal supplement of two-process unravelings because it can be interpreted in terms of pairs of base vectors.

The goal of developing a new simulation methodology for quantum field theory based on two-process unravelings combined with the Bloch equation was a main motivation for the present work. Such simulations are fundamentally different from the widely used simulations based on Wilson's famous formulation of lattice gauge theories [30-32]. Computer simulations of lattice gauge theories with dynamic fermions $[33,34]$ have been established as a very successful tool in nonperturbative quantum field theory, but they are extremely demanding from a computational point of view. Our starting point for an alternative simulation methodology is the dissipative version of quantum field theory initiated in [35] and fully developed in [23], which is based on a thermodynamic quantum master equation. The major challenge for the new simulations is to develop proper importance sampling schemes for high-dimensional problems. (In a rudimentary form, this has already been done for quantum electrodynamics in [36].) Our two-level system can be interpreted as a toy version of scalar field theory ( $\varphi^{4}$ theory). The free ground state corresponds to the vacuum state of the free field theory, and the excited state contains four free particles with total mass 2 , where momentum effects are negligible. With this interpretation, the operators $A^{\dagger}$ and $A$ create and annihilate four free particles. The vacuum state of the interacting theory differs from the free vacuum state by a contribution from the four-particle state, lowering the energy. The matrix element shown in Fig. 5 can be interpreted as a four-particle propagator.

A special feature of the thermodynamic quantum master equation (6) is that the coupling operators $A, A^{\dagger}$ are adapted to the free theory [see Eq. (4)] rather than to the full interacting theory. This is the origin of nonlinearity in the thermodynamic quantum master equation, which can only be avoided if the coupling operators correspond to jumps between the energy eigenstates of the full theory [5]. In dissipative quantum field theory, coupling operators causing transitions between free states are meaningful because the particles of the interacting theory are clouds of free particles and dissipation is supposed to smear out the free constituents so that the clouds become unresolvable. This separate relevance of the free theory (independent of perturbation theory or the use of the interaction picture) leads to the nonlinearity of the quantum master equation of dissipative quantum field theory [23]. This nonlinearity of the quantum master equation at finite temperatures explains why simulations of quantum field theory, which involve a huge number of degrees of freedom, should be based on the simpler zero-temperature quantum master equation investigated in the present paper. While nonlinear quantum master equations are controversial and usually not required, the search for robust equations in dissipative quantum field theory might actually provide a convincing motivation for studying these nonlinear equations.

\section{ACKNOWLEDGMENT}

I am grateful to Martin Kröger and Maksym Osmanov for many stimulating discussions.

\section{APPENDIX: THERMODYNAMIC QUANTUM MASTER EQUATIONS}

In this Appendix, we sketch the development of quantum master equations arising from the quantization of the theory of classical dissipative systems. As in the classical framework of nonequilibrium thermodynamics [12], we assume that the reversible and irreversible contributions to time-evolution equations are generated by energy and entropy, respectively. In the simplest case of the coupling to a heat bath, a constant temperature $T$ is available and we can combine energy and entropy into a Helmholtz free energy operator,

$$
\mu_{t}=H+k_{\mathrm{B}} T \ln \rho_{t},
$$

where $\rho_{t}$ is the density matrix and $-k_{\mathrm{B}} \ln \rho_{t}$ is the entropy operator (the average of which is the von Neumann entropy). This combined operator generates both the reversible and 
the irreversible terms in the thermodynamic quantum master equation

$$
\begin{aligned}
\frac{d \rho_{t}}{d t}= & -i\left[\mu_{t}, \rho_{t}\right]-\int_{0}^{1} f(u)\left(\left[A, \rho_{t}^{1-u}\left[A^{\dagger}, \mu_{t}\right] \rho_{t}^{u}\right]\right. \\
& \left.+\left[A^{\dagger}, \rho_{t}^{u}\left[A, \mu_{t}\right] \rho_{t}^{1-u}\right]\right) d u,
\end{aligned}
$$

with the coupling operators $A, A^{\dagger}$ and the non-negative rate function $f(u)$. The reversible term is generated by a single commutator, whereas the irreversible term is generated by double commutators; note the analogy to first- and second-order derivatives in Fokker-Planck equations. The irreversible term is written such that $\rho_{t}$ remains self-adjoint for any real function $f(u)$. For constant $\mu_{t}$, we obtain the steady-state solution of Eq. (A2). According to Eq. (A1), the constant $\mu_{t}$ corresponds to the Gibbs state $\rho_{\mathrm{eq}}$, where the normalization of the density matrix determines the value of that constant. Equation (A2), moreover, guarantees non-negative entropy production. For finite-dimensional systems, it has been argued in [5] that the singular behavior of the entropy operator $-k_{\mathrm{B}} \ln \rho_{t}$ for an eigenvalue of the density matrix approaching zero implies that the density matrix must remain non-negative to be consistent with non-negative entropy production.
The property (4) implies the identity

$$
\begin{aligned}
\frac{d}{d u}\left(e^{-2 \beta u} \rho^{u} A \rho^{1-u}\right) & =-e^{-2 \beta u} \rho^{u}(2 \beta A+[A, \ln \rho]) \rho^{1-u} \\
& =-e^{-2 \beta u} \rho^{u}\left[A, \beta H^{\text {free }}+\ln \rho\right] \rho^{1-u}
\end{aligned}
$$

which, together with its adjoint, allows us to rewrite Eq. (A2) for $f(u)=\beta \gamma e^{-2 \beta u}$ in the form given in Eq. (6). We thus realize why the property (4) together with a properly matched choice of $f(u)$ leads to a linear master equation for the free theory.

Although Eq. (A2) is convenient for linearization around equilibrium (because the operator $\mu_{t}$ is small except for an irrelevant constant), we here prefer to linearize the equivalent equation (6). If we subtract the equilibrium version of Eq. (6), we need to evaluate terms of the form $\rho_{t}^{u}-\rho_{\mathrm{eq}}^{u}$ in terms of $\Delta \rho_{t}$. In general, this is nontrivial. In the limit of vanishing temperature, however, $2 \beta e^{-2 \beta u}$ approaches a Dirac $\delta$ function so that we need to evaluate $\rho_{t}^{u}-\rho_{\mathrm{eq}}^{u}$ only at $u=1$ or $u=0$, with the trivial results $\Delta \rho_{t}$ and 0 , respectively. We then arrive at the zero-temperature quantum master equation (10).
[1] H.-P. Breuer and F. Petruccione, The Theory of Open Quantum Systems (Oxford University Press, Oxford, 2002).

[2] U. Weiss, Quantum Dissipative Systems, 3rd ed, Series in Modern Condensed Matter Physics (World Scientific, Singapore, 2008), Vol. 13.

[3] G. Lindblad, Commun. Math. Phys. 48, 119 (1976).

[4] H. C. Öttinger, Europhys. Lett. 94, 10006 (2011).

[5] D. Taj and H. C. Öttinger, Phys. Rev. A 92, 062128 (2015).

[6] M. Grmela, Phys. Lett. A 102, 355 (1984).

[7] A. N. Kaufman, Phys. Lett. A 100, 419 (1984).

[8] P. J. Morrison, Phys. Lett. A 100, 423 (1984).

[9] P. J. Morrison, Phys. D (Amsterdam, Neth.) 18, 410 (1986).

[10] M. Grmela and H. C. Öttinger, Phys. Rev. E 56, 6620 (1997).

[11] H. C. Öttinger and M. Grmela, Phys. Rev. E 56, 6633 (1997).

[12] H. C. Öttinger, Beyond Equilibrium Thermodynamics (Wiley, Hoboken, NJ, 2005).

[13] E. B. Davies, Commun. Math. Phys. 39, 91 (1974).

[14] J. Flakowski, M. Osmanov, D. Taj, and H. C. Öttinger, Europhys. Lett. 113, 40003 (2016).

[15] H. Grabert, Z. Phys. B 49, 161 (1982).

[16] H. Grabert, Projection Operator Techniques in Nonequilibrium Statistical Mechanics (Springer, Berlin, 1982).

[17] H. Grabert and P. Talkner, Phys. Rev. Lett. 50, 1335 (1983).

[18] P. Talkner, Ann. Phys. (NY) 167, 390 (1986).

[19] R. Karrlein and H. Grabert, Phys. Rev. E 55, 153 (1997).

[20] H. Grabert, Chem. Phys. 322, 160 (2006).

[21] J. Flakowski, M. Osmanov, D. Taj, and H. C. Öttinger, Phys. Rev. A 90, 042110 (2014).
[22] H. C. Öttinger, Phys. Rev. A 82, 052119 (2010).

[23] H. C. Öttinger, A Philosophical Approach to Quantum Field Theory (Cambridge University Press, Cambridge, UK, 2017).

[24] C. W. Gardiner and P. Zoller, Quantum Noise: A Handbook of Markovian and Non-Markovian Quantum Stochastic Methods with Applications to Quantum Optics, 3rd ed., Springer Series in Synergetics (Springer, Berlin, 2004), Vol. 56.

[25] N. S. Blunt, T. W. Rogers, J. S. Spencer, and W. M. C. Foulkes, Phys. Rev. B 89, 245124 (2014).

[26] F. D. Malone, N. S. Blunt, J. J. Shepherd, D. K. K. Lee, J. S. Spencer, and W. M. C. Foulkes, J. Chem. Phys. 143, 044116 (2015).

[27] R. Kubo, M. Toda, and N. Hashitsume, Nonequilibrium Statistical Mechanics, 2nd ed., Statistical Physics (Springer, Berlin, 1991), Vol. II.

[28] H. C. Öttinger, Phys. Rev. A 86, 032101 (2012).

[29] J. Flakowski, M. Schweizer, and H. C. Öttinger, Phys. Rev. A 86, 032102 (2012).

[30] K. G. Wilson, Phys. Rev. D 10, 2445 (1974).

[31] M. Creutz, Phys. Rev. D 21, 2308 (1980).

[32] H. J. Rothe, Lattice Gauge Theories: An Introduction, 4th ed., World Scientific Lecture Notes in Physics (World Scientific, Hackensack, NJ, 2012), Vol. 82.

[33] S. Duane and J. B. Kogut, Nucl. Phys. B 275, 398 (1986).

[34] S. Gottlieb, W. Liu, D. Toussaint, R. L. Renken, and R. L. Sugar, Phys. Rev. D 35, 2531 (1987).

[35] H. C. Öttinger, Phys. Rev. D 84, 065007 (2011).

[36] H. C. Öttinger, Phys. Rev. D 90, 085005 (2014). 